\title{
Ist unter Ustekinumab das kardiovaskuläre Risiko erhöht?
}

Möglicherweise gibt es eine Assoziation zwischen der Behandlung mit Ustekinumab und einem erhöhten Risiko für akutes Koronarsyndrom und Schlaganfall bei kardiovaskulär vorbelasteten Patienten, das legt eine französische Studie nahe.

\begin{abstract}
Ustekinumab wird unter anderem zur Behandlung von mittelschwerer bis schwerer Psoriasis eingesetzt. Die bisherige Studienlage zu möglichen Effekten der Therapie auf die kardiovaskuläre Gesundheit ist kontrovers. Französische Forscher entdeckten jetzt Hinweise darauf, dass die Gabe des Interleukin-12/23-Inhibitors mit einem erhöhten Risiko für akutes Koronarsyndrom und Schlaganfall assoziiert sein könnte, wenn die Patienten bereits ein hohes kardiovaskuläres Grundrisiko haben [1].
\end{abstract}

\section{Die Studie im Detail}

Für die Fall-Kontroll-Studie werteten die Wissenschaftler Krankenversicherungsdaten von knapp 9300 Patienten aus, die Ustekinumab erhalten hatten, und teilten sie in zwei Gruppen mit hohem und niedrigem kardiovaskulären Grundrisiko. Wenn ein kardiovaskuläres Ereignis stattgefunden hatte, definiert als

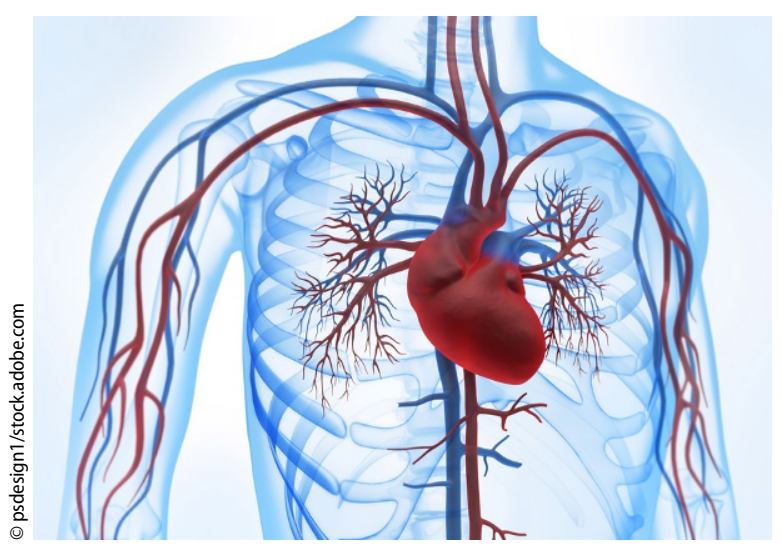

Abb. 1 \ Möglicherweise könnte die durch die Behandlung ausgelöste Hemmung von Typ 17-T-Helferzellen mit einer Destabilisierung von atherosklerotischen Plaques assoziiert sein

\section{Das Wichtigste in Kürze}

Ist das Risiko für schwere kardiovaskuläre Ereignisse nach Beginn der Behandlung mit dem Interleukin-12/23-Inhibitor Ustekinumab erhöht?

Die Studie legt nahe, dass die Behandlung von kardiovaskulär vorbelasteten Patienten mit Ustekinumab mit einem erhöhten Risiko für akutes Koronarsyndrom oder Schlaganfall assoziiert ist.

Bedeutung: Die Studienautoren raten, Ustekinumab bei Patienten mit hohem kardiovaskulärem Grundrisiko mit Vorsicht zu verschreiben.

Einschränkung: Es handelt sich um eine Beobachtungsstudie, eine Kausalität des Zusammenhangs ist nicht belegt.

akutes Koronarsyndrom oder Schlaganfall, wurden die sechs vorausgegangen Monate als Risikozeitraum definiert. Er wurde mit der Referenzzeit von sechs Monaten vor dem Risikozeitraum verglichen.

\section{Kein Effekt bei Patienten mit niedrigem Risiko}

Insgesamt traten 179 kardiovaskuläre Ereignisse auf. Bei den Hochrisikopatienten war die Wahrscheinlichkeit dafür nach dem Behandlungsbeginn mit Ustekinumab viermal so hoch (OR $4,17)$ wie vorher, beobachteten Dr. Florence Poizeau von der Universität Rennes und Kollegen. Im Gegensatz dazu konnten sie bei den Patienten mit niedrigem Grundrisiko keine signifikante Assoziation feststellen.

Die Wissenschaftler vermuten, dass die durch die Behandlung ausgelöste Hemmung von Typ 17-T-Helferzellen mit einer Destabilisierung von atherosklerotischen Plaques assoziiert sein könnte (• Abb. 1). Da es sich jedoch um eine Beobachtungsstudie ohne Nachweis eines kausalen Zusammenhangs handelt, sollten die Ergebnisse vorsichtig interpretiert werden.
„Die Studie legt nahe, dass Vorsicht geboten ist, wenn Ustekinumab Patienten mit hohem kardiovaskulärem Risiko verschrieben wird. Dies scheint eher Personen mit Psoriasis als mit Morbus Crohn zu betreffen, wahrscheinlich weil sie älter sind und ein höheres kardiovaskuläres Risiko haben", so Poizeau und Kollegen.

\section{Literatur}

1. Poizeau F et al (2020) Association Between Early Severe Cardiovascular Events and the Initiation of Treatment With the Anti-Interleukin 12/23p40 Antibody Ustekinumab. JAMA Dermatol. https://doi.org/10.1001/ jamadermatol.2020.2977

Hinweis des Verlags. Der Verlag bleibt in Hinblick auf geografische Zuordnungen und Gebietsbezeichnungen in veröffentlichten Karten und Institutsadressen neutral.

hautnah $2021 \cdot 20: 28$

https://doi.org/10.1007/s12326020-00414-6

(c) Springer-Verlag GmbH Austria, ein Teil von Springer Nature 2020 